\section{Mitem, una propuesta de marco para la integración de las tecnologias en la educación musical.}

UTE. Revista de Ciències de l'Educació

2018 núm. 1. Pag. 20-31

ISSN 1135-1438. EISSN 2385-4731

http://revistes.publicacionsurv.cat/index.php/ute

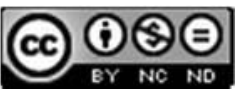

DOI: https://doi.org/10.17345/ute.2018.1

\author{
Eduard Masdeu Yélamos
}

Rebut: 19/10/2017 Acceptat: 08/07/2018

\title{
Resumen
}

La presencia de las tecnologías, si bien provocan mucho interés en los maestros por las amplias posibilidades pedagógicas que ofrecen como herramientas de aprendizaje, todavía son -dentro de la educación musical- un recurso infrautilizado en muchos centros educativos. En este artículo se presenta MITEM, una propuesta de marco para la integración de las tecnologías en la educación musical que identifica 4 factores y 13 elementos que mediatizan el uso de las tecnologías en la enseñanza de la música en la etapa de Educación Primaria con el propósito de que los maestros puedan incorporarlas adecuadamente en los procesos musicales.

Palabras clave: Educación primaria, Maestro de música, Aula de música, Tecnología, Integración curricular.

\begin{abstract}
Educational technology, while generating a great deal of interest among teachers for the huge potential they offer as teaching tools, is still -in music education- an underused resource in many schools. In this article, a brief description of FITME, a general framework proposal for integrating technology in music education, will be provided. FITME identifies 4 factors and 13 elements which facilitate the use of technology in primary school music education, with the aim that music teachers use technologies in teaching in a conscious way.
\end{abstract}

Keywords: Primary education, Primary music specialist teacher, Music classroom, Technology, Curricular integration. 


\section{Introducción}

La irrupción de las tecnologías en el ámbito educativo está generando una eclosión de cambios que, indefectiblemente, "dan un giro histórico a todo lo que hace referencia a las instituciones educativas, desde el currículo y la didáctica hasta la organización y las relaciones personales entre los diferentes agentes del acto educativo" (López, 2002, p.10). A pesar de que las instituciones educativas no se han distinguido por ser innovadoras, las escuelas del siglo XXI no pueden obviar que "las tecnologías forman parte del mundo actual" (Duro \& Aguerrondo, 2008, p.147), de manera que si la sociedad cambia, las escuelas se deben ajustar a las nuevas exigencias que plantea la sociedad e incorporarlas en las aulas con el fin de favorecer la alfabetización digital de los alumnos.

\section{El reto de la integración efectiva de las tecnologías en las aulas de música}

Desde un punto de vista histórico, desde principios del siglo pasado hasta la actualidad muchas escuelas han ido incorporando diversas innovaciones tecnológicas de una forma más o menos anecdótica en las aulas. Sin embargo, no ha sido hasta la implantación de la Ley Orgánica de Educación -que contiene referencias explícitas al desarrollo y aplicación de las tecnologías en los procesos educativos en las etapas educativas obligatorias- cuando verdaderamente la mayoría de las escuelas las han integrado dentro de los procesos de enseñanza y aprendizaje. A la vez, y con distinta intensidad y convicción, los diferentes gobiernos han impulsado programas institucionales de digitalización de las aulas con el objetivo de favorecer la alfabetización digital de los alumnos y mejorar la calidad y la eficacia de los aprendizajes escolares.

Si nos centramos en la enseñanza de la música, este proceso de implementación y utilización de las tecnologías dentro del aula abre un abanico de nuevas oportunidades en los procesos educativos musicales, tanto a nivel de práctica docente como de aprendizaje de los alumnos. En este contexto de despliegue y aplicación de las tecnologías en las aulas de música, diversos autores han enumerado una serie de motivos por los cuales las escuelas deben potenciar el uso de las tecnologías en la enseñanza de música:

- Pueden mejorar las prácticas docentes, enriquecer la experiencia musical dentro del aula y facilitar las tareas de gestión y planificación didáctica que lleva a cabo el maestro de música (Torres, 2011).

- Permiten ampliar el tiempo relativo a los procesos de instrucción musicales, implicar el alumnado en procesos de instrucción dirigidos y la posibilidad que cada alumno trabaje en función de su propio ritmo de aprendizaje (Kassner, 2010).

- Ayudan a mejorar los procesos de aprendizaje musicales (King, 2006; Merrick, 2009).

- Fomentan el aprendizaje activo, la construcción del conocimiento, la investigación y la exploración por parte del alumno (Graesser, Chipman y King, 2008, p.211).

- Favorecen que la enseñanza de la música no se desvincule de las nuevas formas de hacer y escuchar música de nuestra sociedad (Fuertes, 1997).

- La entrada de medios tecnológicos que combinan audio, gráficos y vídeo en un mismo soporte ofrecen la sensación tanto al docente como en el alumno de convertir el proceso de enseñanza y aprendizaje musical en un juego (Bautista, 2004).

- Fomentan la creatividad, la imaginación y la innovación musical del alumnado (Savage, 2005).

Ahora bien, aun teniendo en cuenta las potencialidades educativas que pueden ofrecer las tecnologías como herramientas de aprendizaje, es un error pensar que por el solo hecho de incorporarlas se mejora la calidad y la eficacia de los aprendizajes y se favorece la capacitación del alumnado en la adquisición de los conocimientos, habilidades y actitudes en el ámbito de las competencias digitales. Dicho de otra manera, la inclusión de las tecnologías es un proceso complejo en el que intervienen una serie factores que condicionan la forma en que se integran dentro del aula, de manera que tan solo su implementación tendrá un impacto significativo en los aprendizajes escolares si son incorporadas de manera reflexiva y planificada. Ateniendo a 
estas consideraciones, el marco que se presenta a continuación puede resultar interesante y de gran utilidad a todos los maestros que desean integrar de manera eficiente las tecnologías en las aulas de música.

\section{Metodología}

\subsection{Objetivo}

MITEM -acrónimo de Marco para la Integración de las Tecnologías en la Educación Musical- es uno de los resultados de la investigación "The Digitalisation Of Music Classrooms In Schools In Catalonia: Study Of And Proposal For A General Framework For Integrating Technology In Music Education" (Masdeu, 2015). Uno de sus objetivos es el de identificar los elementos y factores que mediatizan la implementación de las tecnologías en la enseñanza de la educación musical.

\subsection{Proceso de elaboración del MITEM}

En cuanto al proceso de planeo y elaboración del MITEM se establecen tres fases de trabajo:

- Primera fase: Ideación y diseño. En ella se idea una propuesta inicial de MITEM a partir de la información obtenida de: (a) la aplicación de un cuestionario sobre el estado actual en el que se encuentra la incorporación de las tecnologías en las aulas de música de las escuelas catalanas a los maestros de música que trabajan en las escuelas de Cataluña, (b) la realización de 10 entrevistas a expertos de ámbito nacional en la utilización de las tecnologías en el campo de la educación musical sobre la digitalización de las aulas de música de las escuelas de Cataluña, (c) la revisión bibliográfica y documental de estudios previos sobre la digitalización de las aulas de música y (d) la propia experiencia profesional.

- Segunda fase: Validación de la propuesta inicial. Consiste en la realización de 6 entrevistas a expertos de ámbito internacional en la utilización de las tecnologías en el campo de la educación musical con el objetivo de contrastar la propuesta inicial de MITEM.

- Tercera fase: Revisión y afinación de la propuesta. Consiste en ultimar la propuesta de MITEM a partir de las conclusiones obtenidas de las dos fases anteriores.

\subsection{Instrumentos}

En relación con los instrumentos de recogida de datos, se diseñan y aplican diferentes instrumentos con el propósito de identificar los factores y elementos que mediatizan el uso de las tecnologías en la enseñanza de la educación musical en la etapa de Educación Primaria y que, en consecuencia, hay que considerar en el momento de integrar las tecnologías en las aulas de música. Estos sirven de base para la elaboración del MITEM.

Se diseña y aplica un cuestionario en línea estructurado en 5 dimensiones (caracterización de los centros educativos, perfil del maestro de música, inclusión de las tecnologías en la tarea docente, integración institucional de las tecnologías, y otros comentarios y observaciones) con el fin de caracterizar el estado actual en el que se encuentra la incorporación de las tecnologías en las aulas de música de las escuelas catalanas. Se crea a partir de ítems representativos con el propósito general del instrumento y que provienen de distintos cuestionarios. De este modo se garantiza, por un lado la idoneidad de cada uno de los ítems que configuran el instrumento de evaluación (Mertens, 2005; citado a Hernández, Fernández y Baptista, 2010) y del otro la validez del instrumento a través de la representatividad conceptual (Hernández, Fernández y Baptista, 2010). Se desestima analizar la fiabilidad del cuestionario atendiendo que: (a) es un instrumento que se construye de manera específica con el propósito de poder ofrecer una visión puntual del estado actual en que se encuentra la incorporación de las tecnologías en las aulas de música en las escuelas de Cataluña, (b) no pretende ser un instrumento generalizable para otras investigaciones que tengan como objetivo el estudio de la digitalización de las aulas de música y (c) no se dispone de un coeficiente de confiabilidad que permita hacer el cálculo al tratarse de un cuestionario que, mayoritariamente, se compone de ítems que recogen datos nominales. Igualmente, se realiza una prueba interna antes para enviar el cuestionario con el propósito de verificar su correcto funcionamiento. Referente a su aplicación, se establece un procedimiento común con el objetivo de que todas las escuelas tengan las mismas condiciones al completar el cuestionario. Los datos fueron recolectados durante un período de dos meses (de octubre a noviembre de 2011). El cuestionario fue 
enviado a 1.371 escuelas, de las cuales el $35.1 \%$ respondieron. Los datos obtenidos a partir de las preguntas de la encuesta se analizaron utilizando estadísticas descriptivas.

En cuanto a las entrevistas, se diseñan dos modelos de entrevistas semiestructuradas, una para los expertos de ámbito nacional con la que se pretende: (a) obtener una visión complementaria sobre la digitalización de las aulas de música en las escuelas catalanas y (b) entrever si las estrategias declaradas por los especialistas de música en los cuestionarios se aplican, y otra para los expertos de ámbito internacional con la que se pretende: (a) obtener una visión complementaria sobre el proceso de implementación y convergencia de las tecnologías en las aulas de música y (b) contrastar la propuesta de MITEM ideada en la primera fase de trabajo. Se desestima validar el guion de las entrevistas atendiendo que su propósito principal es el de recoger una visión complementaria sobre la digitalización de las aulas de música, y no el de ser un instrumento para la práctica profesional de los especialistas de música. Referente a la aplicación de las entrevistas, destacar que participan 16 expertos de ámbito nacional e internacional en la utilización de las tecnologías aplicadas al campo de la educación musical. Se seleccionan mediante un sistema de muestreo intencional en función de dos criterios: (a) que sean personas referentes en la utilización de las tecnologías aplicadas al campo de la educación musical y (b) que sean personas conocedoras del estado actual en que se encuentra la incorporación de las tecnologías en el aula de música de las escuela de Cataluña (únicamente en el caso de los expertos de ámbito nacional). Los datos obtenidos de las entrevistas se analizaron mediante un análisis cualitativo.

\section{Presentación del MITEM}

El diseño del MITEM es el resultado de tener en cuenta la legislación que regula la ordenación curricular de la enseñanza de la educación musical en la etapa de Educación Primaria en Cataluña, las estrategias que promueven las administraciones educativas para impulsar el uso de las tecnologías en las aulas escolares (Generalitat de Catalunya, 2013; Benavides \& Pedró, 2007; Pelgrum \& Law, 2004), los modelos tecnológicos de implementación de las tecnologías en las escuelas que proponen Newhouse, Trinidad y Clarkson (2002), Marquès (2000a) y Radencich, McKay y Paratore (1995); estudios previos relacionados con: (a) el proceso de implementación y convergencia de las tecnologías en los centros educativos (Lugo \& Kelly, 2011; Generalitat de Catalunya, 2010; Pablos, Colás \& González, 2010; ISTE, 2008; Morrissey 2008; Brunner, 2008; Hew \& Brush, 2007; Tejedor \& García-Valcárcel, 2006; Área, 2005; UNESCO, 2004; BECTA, 2004; Aviram, 2003; UNESCO, 2002), (b) el proceso de integración de las tecnologías en la práctica docente (Koehler \& Mishra, 2009; UNESCO, 2008; ISTE, 2008; Jones, 2007; Perrenoud, 2007; Marquès, 2000b) y (c) el proceso de integración de las tecnologías en la enseñanza de la música (Pérez, 2011; Figueras, 2009; PRELUDE, 2007). 


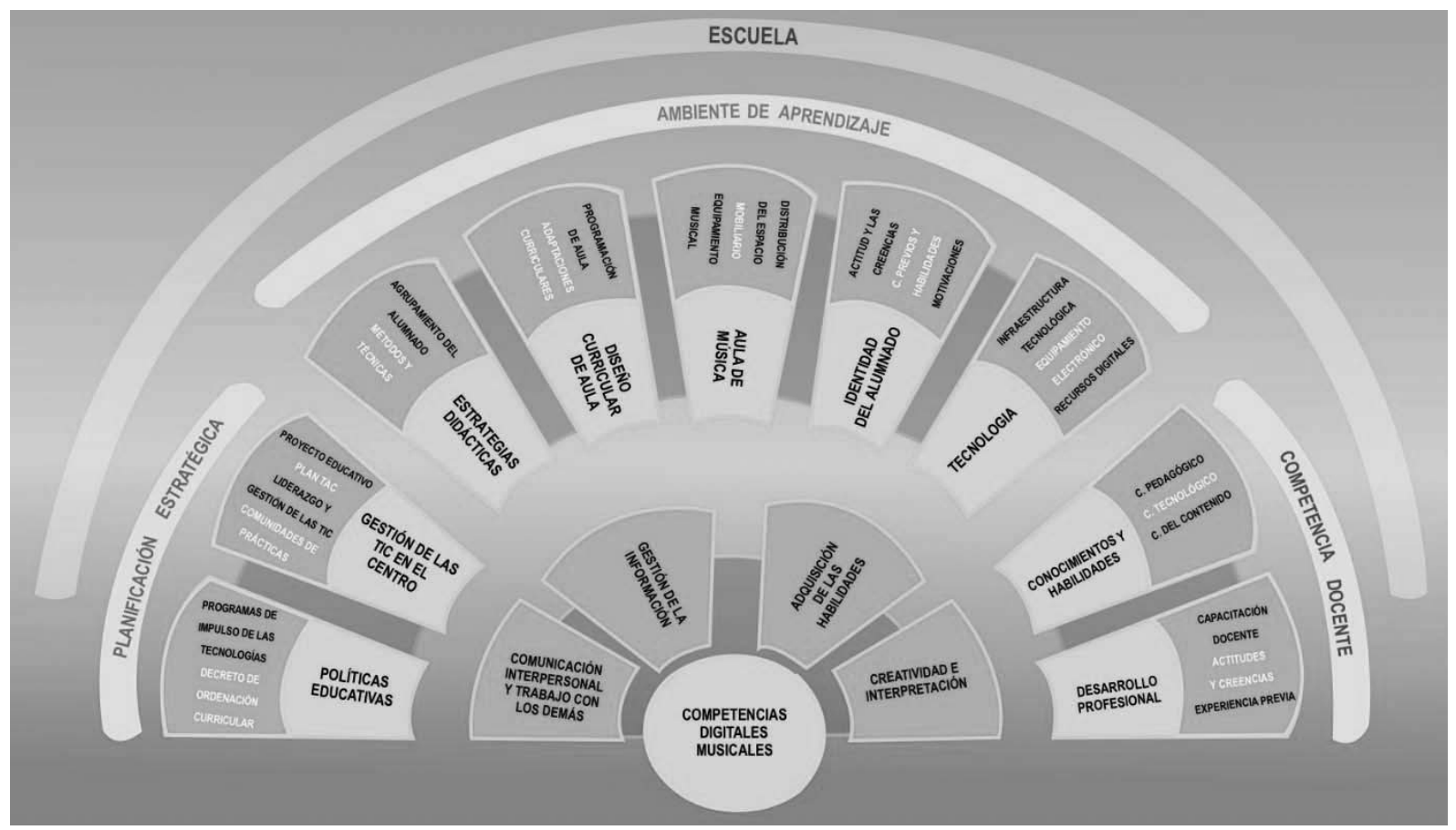

Figura 1: MITEM

MITEM sitúa cuatro factores esenciales que deben considerarse en el momento de integrar las tecnologías en la enseñanza de la educación musical en la etapa de Educación Primaria (ver Figura 1): las competencias digitales musicales (apartado 4.1.), la planificación estratégica (apartado 4.2.), el ambiente de aprendizaje (apartado 4.3.) y la competencia docente (apartado 4.4.). Respecto a la posición de los factores en el diseño de la representación, el marco consta de un núcleo principal que corresponde al primer factor, el de las competencias digitales musicales. Este núcleo se ve influenciado directamente por tres núcleos secundarios que corresponden a los factores de la planificación estratégica, el ambiente de aprendizaje y la competencia docente, situados todos ellos alrededor del núcleo principal. Asimismo, los cuatro factores quedan aglutinados por el contexto de la escuela. Se ha optado por situar a las competencias digitales musicales como núcleo principal del marco teniendo en cuenta que el propósito de la integración de las tecnologías en los procesos de enseñanza y aprendizaje es el de estar "al servicio del aprendizaje y el conocimiento" (Generalitat de Catalunya, 2010, p.5).

\subsection{Primer factor: las competencias digitales musicales}

En el primer factor quedan situadas cuatro competencias que hacen referencia a objetivos educativos musicales que incluyen la utilización de las tecnologías y que favorecen, además de la adquisición de las competencias propias del área de música, la capacitación del alumnado en la adquisición de los conocimientos, habilidades y actitudes en el ámbito de las competencias digitales.

- La primera competencia, comunicación interpersonal y trabajo con los demás, comprende los procesos de trabajo basados en el intercambio de experiencias, sentimientos y/o emociones entre personas o grupos de trabajo: comentar, compartir, participar, transmitir y utilizar. Un ejemplo de actividad que promueve esta primera competencia consiste en crear un patrón rítmico de 4 compases con la ayuda del editor Noteflight y compartir el resultado en el foro del aula virtual.

- La segunda competencia, gestión de la información, incluye las tareas relacionadas con la búsqueda, manipulación y tratamiento del lenguaje: acceder, analizar, buscar, clasificar, almacenar, enlazar, indagar, resumir y usar. Un ejemplo de actividad es analizar el ritmo y la melodía de una canción con la ayuda de MusicTime.

- La tercera competencia, adquisición de las habilidades, comprende las tareas relacionadas con la percepción, conocimiento y comprensión de los elementos propios del lenguaje musical: analizar, evaluar, clasificar, comparar, discriminar, escuchar, leer, memorizar, percibir, reconocer, recordar y usar. Un ejemplo de actividad es evaluar el conocimiento del alumnado sobre los instrumentos de la orquestra 
con la ayuda de un cuestionario digital (http://www.xtec.cat/trobada/musica/jocs/pregunt/question.htm)

- La cuarta competencia, creatividad e interpretación, incluye las tareas relacionadas con la expresión y la experimentación de las posibilidades creativas del hecho sonoro: aplicar, comprender, crear, ejecutar, experimentar, improvisar, indagar y utilizar. Un ejemplo de actividad que promueve esta cuarta competencia consiste en crear sencillas producciones musicales colectivas mediante la aplicación Toc and Roll.

\subsection{Segundo factor: la planificación estratégica}

En el segundo factor quedan situados los elementos vinculados con la estructura normativa y de gestión del centro educativo: las políticas educativas y la gestión de las TIC en el centro.

El primer elemento, políticas educativas, identifica los aspectos que hacen referencia a las estrategias de las administraciones educativas en relación a la incorporación de las tecnologías en las escuelas: los programas de impulso de las tecnologías y el decreto de ordenación curricular.

- Programas de impulso de las tecnologías. Desde la llegada de los ordenadores personales y de la enseñanza asistida por ordenador en el mundo de la educación, la mayor parte de los gobiernos locales, regionales y/o nacionales de todo el mundo han incorporado en sus políticas educativas proyectos relacionados con la integración de las tecnologías en las escuelas (OECD, 2009). Como muestra, destacamos el programa Escuela 2.0, el Proyecto EduCAT 2.0 y el plan de Cultura Digital en la Escuela.

- Decreto de ordenación curricular. De acuerdo con las competencias compartidas que dispone la Generalitat de Cataluña para el establecimiento de los planes de estudio correspondientes a la educación obligatoria, corresponde al gobierno de la Generalitat regular la ordenación curricular de la Educación Primaria teniendo en cuenta las enseñanzas mínimas establecidas por el Gobierno. Así pues, en el decreto 142/2007 del 26 de junio se precisan las orientaciones metodológicas, las competencias básicas, los objetivos, los contenidos y los criterios de evaluación correspondientes al conjunto de la etapa de Educación Primaria y a cada una de las áreas que la integran -entre ellas, la educación artística.

El segundo elemento, gestión de las TIC en el centro, recoge aquellos aspectos que deben contemplarse en lo referente a la gobernanza de las tecnologías en la escuela: el proyecto educativo, el Plan TAC, el liderazgo y la gestión de las tecnologías, y las comunidades de prácticas.

- El proyecto educativo. De acuerdo con el decreto 142/2007 del 26 de junio, cada centro educativo debe elaborar un documento -llamado proyecto educativo- con el fin de desarrollar y concretar el currículo de Educación Primaria según las características del alumnado presente en la escuela.

- El Plan TAC. Informes como los de Lugo y Kelly (2011) o de la Generalitat de Catalunya (2010) señalan la importancia de que el proceso de integración de las tecnologías en las escuelas sea fruto de un proceso de reflexión y planificación consensuado entre todo el claustro docente. El Plan TAC es un documento de centro -en Cataluña promovido por el Departament d'Ensenyament y dirigido a todos los centros educativos- en el que se recoge la madurez digital de la escuela y se concretan actuaciones que hacen referencia a aspectos organizativos, didáctico-pedagógicos y curriculares con el objetivo de asegurar la competencia digital del alumnado, la integración curricular de las tecnologías, la inclusión digital y la innovación metodológica (Generalitat de Catalunya, 2010).

- El liderazgo y la gestión de las tecnologías. Hay que fomentar un clima escolar que incentive a los docentes a experimentar con las tecnologías (Bitner \& Bitner, 2002). Para ello, es primordial que el equipo directivo -con la colaboración de un equipo de soporte TIC- impulse acciones estratégicas que asesoren y motiven al resto del claustro a integrar las tecnologías en los procesos de enseñanza y aprendizaje.

- Las comunidades de prácticas. Son grupos de docentes que se reúnen para compartir problemas y/o consejos en torno a un tema específico -en este caso, la utilización de las tecnologías- así como también para ampliar sus conocimientos a través de la interacción continua entre todos los participantes. La participación docente en estos espacios motivará a que la figura docente gane confianza y seguridad en el uso de las tecnologías. 


\subsection{Tercer factor: el ambiente de aprendizaje}

En el tercer factor quedan situados los elementos relativos a la planificación de la acción formativa dentro de las aulas de música: las estrategias didácticas, el diseño curricular de aula, el aula de música, la identidad del alumnado y la tecnología.

El primer elemento, estrategias didácticas, comprende los aspectos vinculados a pautas de actuación sistemáticas y sistémicas que el maestro puede incorporar en los procesos de enseñanza con el propósito de poder configurar aprendizajes que impliquen el uso de las tecnologías: el agrupamiento del alumnado, y los métodos y técnicas.

- Agrupamiento del alumnado. Según Somekh y Davis (1997), la competencia docente en el uso de las tecnologías no es condición suficiente para integrar las tecnologías en los procesos de enseñanza y aprendizaje. A este respecto, Newhouse (1999) sugiere considerar las diferentes modalidades de organización del grupo clase que se pueden configurar en los procesos de enseñanza y aprendizaje y que favorecen la utilización de las tecnologías.

- Los métodos y técnicas. De acuerdo con Newhouse, Trinidad y Clarkson (2002) podemos distinguir tres modelos básicos de utilización de las tecnologías en los procesos de enseñanza: (a) individual, (b) agrupamiento flexible y (c) con todo el grupo clase. A partir de los tres modelos identificados, se pueden adoptar multiplicidad de técnicas y métodos que favorecen la utilización de las tecnologías en los aprendizajes.

El segundo elemento, diseño curricular de aula, incluye los aspectos que inciden en el proceso de concreción curricular que realiza el maestro a partir de la información incluida en el Proyecto Educativo de Centro: la programación de aula y las adaptaciones curriculares.

- Programación de aula. De acuerdo con Ander-Egg (1993) es el instrumento que permite al maestro organizar y articular su práctica educativa conforme a las finalidades expresadas en el Proyecto Educativo de Centro. Entre los diversos elementos que debe recoger la programación, destacamos: los objetivos didácticos, los contenidos, la metodología, la temporalización, las diferentes actividades de aprendizaje, los materiales y recursos, y los criterios y momentos de evaluación.

- Adaptaciones curriculares. Consiste en adecuar el currículo a las necesidades educativas específicas del alumno con el propósito de que éste pueda alcanzar los objetivos establecidos. Las adaptaciones pueden ser no significativas, si las modificaciones establecidas no requieren adecuar las capacidades fijadas en los objetivos de la materia curricular; o significativas, si las modificaciones establecidas en la adaptación requieren ajustar los criterios de evaluación y el grado de logros de las capacidades del área.

El tercer elemento, el aula de música, aglutina los aspectos que tienen vinculación directa con la organización y configuración del aula específica de educación musical: la distribución del espacio, el mobiliario y el equipamiento musical.

- Distribución del espacio y el mobiliario. Radcliffe, Wilson, Powell y Tibbetts (2008) concluyen que el diseño y el equipamiento del aula condicionan los patrones de enseñanza y aprendizaje que se pueden impulsar dentro de ella. Así pues, a fin de que los docentes puedan desarrollar diversos estilos de enseñanza, es importante que el aula tenga una distribución flexible del espacio y un mobiliario que posibilite diversas configuraciones del espacio.

- Equipamiento musical. Al margen de las especificaciones físicas del espacio y del mobiliario existente dentro del aula, hay que prestar también atención al equipamiento musical que dispone el aula y precisar los instrumentos que pueden ser utilizados en los procesos de enseñanza y aprendizaje.

El cuarto elemento, identidad del alumnado, engloba los aspectos que permiten identificar los rasgos distintivos que particularizan el alumnado: la actitud y las creencias, los conocimientos previos y las habilidades, y las motivaciones.

- El conocimiento de aspectos que singularizan el alumnado en relación al uso de las tecnologías -como son, por ejemplo, la actitud, las creencias, los conocimientos previos y las habilidades o la motivaciónpermiten que el maestro pueda planear y adecuar el uso de éstas en función de las características del alumno. 
Finalmente, el quinto elemento, tecnología, identifica los aspectos relativos al equipamiento tecnológico que dispone el aula: la infraestructura tecnológica, el equipamiento electrónico y los recursos digitales.

- Es importante tener presente qué infraestructura tecnológica dispone el aula (p.e. conectividad a la red) y qué equipamiento electrónico -tanto de carácter general como específico para la práctica de la músicay recursos digitales puede utilizar el maestro en las actividades de aprendizaje diseñadas (p.e. tableta táctil, teclado MIDI, ordenador, software musical).

\subsection{Cuarto factor: la competencia docente}

En el cuarto factor quedan situados dos elementos que tienen vinculación directa con el perfil profesional del maestro: los conocimientos y habilidades del docente, y el desarrollo profesional.

El primer elemento, conocimientos y habilidades, recoge aquellos aspectos que hacen referencia a las competencias y destrezas que debe poseer el maestro para poder hacer un uso efectivo de las tecnologías en la enseñanza de la educación musical: conocimiento pedagógico, conocimiento tecnológico y conocimiento del contenido.

- La irrupción de las tecnologías en las escuelas ha empujado al maestro de música a actualizar sus conocimientos y habilidades en el uso de las tecnologías con el objetivo de poder aprovechar las amplias posibilidades pedagógicas que ofrecen las tecnologías como herramientas de aprendizaje. Siguiendo esta línea, Mishra y Koehler (2006) sugieren un marco conceptual de conocimientos -denominado como TPACK (Conocimiento Tecnológico Pedagógico del Contenido)- que identifica los tipos de conocimientos y las sinergias que se dan entre ellos y que deben poseer los docentes para poder hacer un uso efectivo de las tecnologías en los procesos de enseñanza.

El segundo elemento, desarrollo profesional, comprende los apartados que determinan la forma en que el maestro de música integra el uso de las tecnologías en su actividad profesional: la capacitación docente, las actitudes y creencias, y la experiencia previa.

- Si bien se ha identificado que uno de los motivos más habituales por el que el maestro decide no utilizar las tecnologías en los procesos educativos es la falta de conocimientos y habilidades específicas en el uso de las tecnologías (Hew \& Brush, 2007); las actitudes, las creencias y las experiencias previas de los docentes también juegan un papel decisivo en el proceso de integración de las tecnologías en las aulas. Así por ejemplo, Ramírez, Cañedo y Clemente (2012) destacan que una actitud positiva del maestro hacia los recursos tecnológicos hará que este tenga una mayor predisposición a integrarlos en su labor docente.

- La formación del profesorado es un instrumento que fomenta la confianza en el uso pedagógico de las tecnologías (Paraskeva, Bouta y Papagianni, 2008).

\section{Conclusiones}

Una mayor presencia y uso de las tecnologías en el aula de música puede aportar grandes beneficios en la enseñanza de la educación musical. No sólo por ser herramientas que abren nuevos escenarios educativos sino también porque pueden mejorar la calidad y la eficacia de los aprendizajes y favorecer la alfabetización digital de los alumnos. Ahora bien, a fin de que su incorporación no se convierta en un mero recurso para ser utilizado en actividades musicales puntuales sino en un instrumento "al servicio del aprendizaje y el conocimiento" (Generalitat de Catalunya, 2010, p.5), es importante que su implementación dentro del aula se lleve a cabo de manera reflexiva y planificada. Desde esta perspectiva, la propuesta de marco presentada (MITEM) identifica los factores y elementos que mediatizan el uso de las tecnologías en la enseñanza de la educación musical en la etapa de Educación Primaria y que, en consecuencia, hay que considerar en el momento de integrar las tecnologías en las aulas de música. 


\section{Referencias Bibliográficas}

Ander-Egg, E. (1993). La planificación educativa: conceptos, métodos, estrategias y técnicas para educadores. Buenos Aires: Magisterio del Río de la Plata.

Área, M. (2005). Tecnologías de la información y comunicación en el sistema escolar. Una revisión de las líneas de investigación. Revista Electrónica de Investigación y Evaluación Educativa, 11, 3-25. Recuperado de https://www.uv.es/RELIEVE/v11n1/RELIEVEv11n1_1.htm

Aviram, A. (2003). ICT in education: Should it necessarily be a case of the recurrent reinvention of the Wheel? En A. Hargreaves \& J. Sancho (Eds.), The Geographics of Educational Change. Londres: Kluwer.

Bautista, J. (2004). Las nuevas tecnologías y la expresión musical, otros lenguajes en la educación. Comunicar, 23, 25-30. Recuperado de https://www.revistacomunicar.com/index.php?contenido $=$ detalles $\&$ numero $=23 \&$ articulo $=23-2004-05$

BECTA. (2004). A review of the research literature on barriers to the uptake of ICT by teachers. British Educational Communications and Technology Agency. Recuperado de http://dera.ioe.ac.uk/1603/1/becta_2004_barrierstouptake_litrev.pdf

Benavides, F. \& Pedró, F. (2007). Políticas educativas sobre nuevas tecnologías en los países iberoamericanos. Revista Iberoamericana de Educación, 45, 19-69. Recuperado de http://rieoei.org/rie45a01.pdf

Bitner, N. \& Bitner, J. (2002). Integrating technology into the Classroom: Eight Keys to Success. Journal of Technology and Teacher Education, 10 (1), 95-100. Recuperado de https://www.learntechlib.org/p/9304/

Brunner, J. (2008). ¿Una sociedad movilizada hacia las TIC? En J.C. Tedesco \& N. Burbules \& otros, Las TIC: del aula a la agenda política. Ponencias del Seminario Internacional. Cómo las TIC transforman las escuelas. Buenos Aires. UNICEF, 2008, 41-53. Recuperado de https://www.unicef.org/argentina/spanish/IIPE_Tic_06.pdf

Duro, E. \& Aguerrondo, I. (2008). Palabras de cierre. En J.C. Tedesco \& N. Burbules \& otros, Las TIC: del aula a la agenda política. Ponencias del Seminario Internacional. Cómo las TIC transforman las escuelas. Buenos Aires. UNICEF, 2008, 143-150. Recuperado de https://www.unicef.org/argentina/spanish/IIPE_Tic_06.pdf

Figueras, M. (2009). Dotacions de suport TAC a l'aula de música: Didàctica de la música al s. XXI. Xarxa Telemàtica Educativa de Catalunya: Llicència d'estudis. Recuperado de http://www.xtec.cat/sgfp/llicencies/200809/memories/1958m.pdf

Fuertes, C. (1997). Educación musical y Tecnologías de la Información y la Cornunicación. Métodos de Información, 4 (21), 32-37. Recuperado de http://eprints.rclis.org/4803/1/1997-21-32.pdf

Generalitat de Catalunya. (2013). Competències bàsiques de l'àmbit digital: identificació $i$ desplegament a l'educació primària. Departament d'Ensenyament. Recuperado de http://ensenyament.gencat.cat/web/.content/home/departament/publicacions/colleccions/competenciesbasiques/primaria/prim-ambit-digital.pdf

Generalitat de Catalunya. (2010). Educació. El pla TAC del centre. Col.lecció TAC-1. Departament d'Educació. Recuperado de http://ensenyament.gencat.cat/web/.content/home/departament/publicacions/colleccions/tac/pla-taccentre/tac_1.pdf 
Graesser, A. C., \& Chipman, P., \& King, B. G. (2008). Computer-mediated technologies En Spector, J. M., Merrill, M. D., \& van Merrienboer, J. J. G., \& Driscoll, M. P (Eds.). Handbook of Research on Educational Communications and Technology. London: Taylor \& Francis.

Hernández, R., Fernández, C., \& Baptista, P. (2010). Metodología de la investigación. México: Mc Graw Hill. Hew, K.F. \& Brush, T. (2007). Integrating technology into K-12 traching and learning: current knowledge gaps and recommendations for future research. Education Tech Research Dev, 55, 223-252. Recuperado de https://doi.org/10.1007/s11423-006-9022-5

ISTE. (2008). Estándares nacionales (EEUU) de tecnologías de información y comunicación (TIC) para docentes. Eduteka. Recuperado de http://eduteka.icesi.edu.co/pdfdir/EstandaresNETSEstudiantes2007.pdf Jones, R. (2007). Learning Technologies and Schools of the future. First BCSE Annual Lecture. London. Bristish Council for School Environment. Recuperado de http://ateneu.xtec.cat/wikiform/wikiexport/_media/materials/jornades/jt101/bloc1/ic8647_bcse_brochure_v7. pdf

Kassner, K. (2010). Using Music Teachnology in the Classroom. Recuperado de https://education.fcps.org/trt/sites/default/files/karen/musictech.pdf

King, A. (2006). Contingent Learning for Creative Music Technologists. Recuperado de http://nrl.northumbria.ac.uk/id/eprint/9791

Koehler, M.J. \& Mishra, P. (2009). What is Technological Pedagogical Content Knowledge? Contemporany Issues in Technological and Teacher Education, 9, 60-70. Recuperado de https://www.learntechlib.org/p/29544/ López, F. (2002). Introducción. En A. Alàs \& A. Bartolomé \& otros, Las Tecnologías de la Información y de la Comunicación en la escuela (pp. 9-12). Barcelona: Editorial Graó.

Lugo, M.T. \& Kelly, V. (2011). La matriz TIC: una herramienta para planificar las Tecnologías de la Información $y$ Comunicación en las instituciones educativas. IIPE - Buenos Aires. Recuperado de http://www.buenosaires.iipe.unesco.org/sites/default/files/Articulo\%2520matriz\%2520TIC_0.pdf

Marquès, P. (2000a). Cambios en los centros educativos: construyendo la escuela del futuro. Recuperado de http://ddd.uab.cat/pub/dim/16993748n0/16993748n0a5.pdf

Marquès, P. (2000b). Los docentes: funciones, roles, competencias necesarias, formación. Recuperado de http://peremarques.net/docentes2.htm

Masdeu, E. (2015). The digitalisation of music classrooms in schools in Catalonia: study of and proposal for a general framework for integrating technology in music education. Universitat de Lleida. Recuperado de http://www.tdx.cat/handle/10803/300305

Merrick, B. (2009). Through the looking glass: Valuing the reflective process to develop understanding in a music technology based environment. ASME XVII National Conference on Musical Understanding. Tasmania: Lauceston.

Mishra, P. \& Koehler, M. (2006). Technological Pedagogical Content Knowledge: A Framework for Teacher Knowledge. Teachers College Record, 108 (6), 1017-1054. Recuperado de https://pdfs.semanticscholar.org/977d/8f707ca1882e093c4ab9cb7ff0515cd944f5.pdf

Morrissey, J. (2008). El uso de TIC en la enseñanza y el aprendizaje. Cuestiones y desafíos. En J.C. Tedesco \& N. Burbules \& otros, Las TIC: del aula a la agenda política. Ponencias del Seminario Internacional. Cómo las TIC 
transforman las escuelas. Buenos Aires. UNICEF, 2008, 81-90. Recuperado de https://www.unicef.org/argentina/spanish/IIPE_Tic_06.pdf

Newhouse, C.P. (1999). Teachers Use Computers in the 21st Century. Perth, Western Australia: Specialist Educational Services.

Newhouse, P. \& Trinidad, S. \& Clarkson, B. (2002). Teacher Professional ICT Attributes: A Framework. Recuperado de http://scosta.pbworks.com/w/file/fetch/51144284/Teacher\%2BICT\%2BAttributes\%2BFramework\%2BUWA

OECD. (2009). Assessing the effects of ICT in education. JRC European Commission. Recuperado de http://dx.doi.org/10.1787/9789264079786-en

Pablos, J. \& Colás, P. \& González, T. (2010). Factores facilitadores de la innovación con TIC en los centros escolares. Un análisis comparativo entre diferentes políticas educativas autonómicas. Revista de Educación, 352, 23-51. Recuperado de http://www.revistaeducacion.educacion.es/re352/re352_02.pdf

Paraskeva, F. \& Bouta, H. \& Papagianni, A. (2008). Individual characteristics and computer self-efficacy in secondary education teachers to integrate technology in educational practice. Computers \& Education, 50, 1084-1091. Recuperado de https://doi.org/10.1016/j.compedu.2006.10.006

Pelgrum, W.J. \& Law, N. (2004). Les TIC et l'éducation dans le monde: tendances, enjeux et perspectives. Paris: Institut international de planification de l'éducation.

Pérez, A. (2011). L'educació Musical i les TAC: Web 2.0 i xarxes socials educatives. Xarxa Telemàtica Educativa de Catalunya: Llicència d'estudis. Recuperado de http://www.xtec.cat/sgfp/llicencies/201011/memories/2083m.pdf

Perrenoud, P. (2007). Diez nuevas competencias para enseñar. Invitación al viaje. Colofón: Graó.

PRELUDE. (2007). Final Report on teacher's needs analysis. Socrates - Comenius. Recuperado de http://www.ea.gr/ep/prelude/material/WP2_final_report.pdf

Radcliffe, D. \& Wilson, H. \& Powell, D. \& Tibbetts, B. (2008). Designing Next Generation Places of Learning: Collaboration at the Pedagogy-Space-Technology Nexus. The University of Queensland. Recuperado de http://citeseerx.ist.psu.edu/viewdoc/download?doi=10.1.1.215.788\&rep=rep1\&type=pdf

Radencich, M.C. \& McKay, L.J. \& Paratore, J.R. (1995). Keeping Flexible Groups Flexible: Grouping Options. En M.C. Radencich y L.J. McKay (Eds.), Flexible Grouping for Literacy in the Elementary Grades. Boston: Allyn and Bacon.

Ramírez, E. \& Cañedo, I. \& Clemente, M. (2012). Las actitudes y creencias de los profesores de secundaria sobre el uso de Internet en sus clases. Revista Comunicar, 38 (19), 147-155. Recuperado de https://doi.org/10.3916/C38-2012-03-06

Savage, J. (2005). Information communication technologies as a tool for re-imagining music education in the 21st century. International Journal of Education \& the Arts, 6 (2).

Somekh, B. \& Davis, N. (Eds.) (1997). Using IT Effectively in Teaching and Learning: Studies in Pre-Service and In-Service Teacher Education. London: Routledge. 
Tejedor, F.J. \& García-Valcárcel, A. (2006). Competencias de los profesores para el uso de las TIC en la enseñanza. Revista Española de Pedagogía, 233 (6), 21-43. Recuperado de https://dialnet.unirioja.es/descarga/articulo/1973261.pdf

Torres, L. (2011). Aplicación de las TIC en el aula de educación musical de la educación primaria: musytic.com, un recurso para el docente. Revista Eufonía, 52, 63-70.

UNESCO. (2008). ICT Competency Standards for Teachers. Implementation Guidelines version 1.0. United Nations Educational, Scientific and Cultural Organization. Recuperado de http://unesdoc.unesco.org/images/0015/001562/156209E.pdf

UNESCO. (2004). Las tecnologías de la información y la comunicación en la formación docente. Organización de las Naciones Unidas para la Educación, la Ciencia y la Cultura. Ediciones. Montevideo: TRILCE.

UNESCO. (2002). Information and communication technology in education. A curriculum for schools and programme of teacher development. United Nations Educational, Scientific and Cultural Organization. Recuperado de http://unesdoc.unesco.org/images/0012/001295/129538e.pdf 